\title{
Extension of Strongly Regular Graphs
}

\author{
Ralucca Gera* \\ Department of Applied Mathematics \\ Naval Postgraduate School, Monterey, CA 93943 \\ email: rgera@nps.edu, phone (831) 656-2206, fax (831) 656-2355 \\ and \\ Jian Shen \\ Department of Mathematics \\ Texas State University, San Marcos, TX 78666 \\ email: js48@txstate.edu, phone: (512) 245-3740
}

Submitted: Sep 30, 2007; Accepted: Feb 2, 2008; Published: Feb 11, 2008

Mathematics Subject Classification: 05C75

\begin{abstract}
The Friendship Theorem states that if any two people in a party have exactly one common friend, then there exists a politician who is a friend of everybody. In this paper, we generalize the Friendship Theorem. Let $\lambda$ be any nonnegative integer and $\mu$ be any positive integer. Suppose each pair of friends have exactly $\lambda$ common friends and each pair of strangers have exactly $\mu$ common friends in a party. The corresponding graph is a generalization of strongly regular graphs obtained by relaxing the regularity property on vertex degrees. We prove that either everyone has exactly the same number of friends or there exists a politician who is a friend of everybody. As an immediate consequence, this implies a recent conjecture by Limaye et. al.
\end{abstract}

Key Words: strongly regular graph, Friendship Theorem

\section{Introduction and Motivation}

In this paper all graphs $G=(V(G), E(G))$ are simple. The neighborhood of a vertex $v \in V(G)$ is $N(v)=\{u:(u, v) \in E(G)\}$. The join, denoted $G_{1} \vee G_{2}$, of two graphs $G_{1}$ and $G_{2}$ is the graph with vertex set $V\left(G_{1}\right) \cup V\left(G_{2}\right)$ and edge set $E\left(G_{1}\right) \cup E\left(G_{2}\right) \cup\{(u, v)$ : $u \in V\left(G_{1}\right)$ and $\left.v \in V\left(G_{2}\right)\right\}$. Also, the disjoint union, denoted by $G_{1}+G_{2}$, of two graphs

\footnotetext{
${ }^{*}$ Research supported by the Research Initiation Program Grant at the Naval Postgraduate School.
} 
$G_{1}$ and $G_{2}$ is the graph obtained from $G_{1}$ and $G_{2}$ with $V\left(G_{1}+G_{2}\right)=V\left(G_{1}\right) \cup V\left(G_{2}\right)$ and $E\left(G_{1}+G_{2}\right)=E\left(G_{1}\right) \cup E\left(G_{2}\right)$.

We use the notation $d(u)=|N(u)|$ and $\delta(u, v)=|N(u) \cap N(v)|$ to denote the number of neighbors of $u$ (the degree of $u$ ) and the number of common neighbors of $u$ and $v$, respectively. An $n$-vertex graph is called strongly regular, denoted $S R G(n, r, \lambda, \mu)$, if there exist nonnegative integers $r, \lambda, \mu$ such that for all vertices $u, v \in V(G)$,

$$
\delta(u, v)= \begin{cases}r & \text { if } u=v ; \\ \lambda & \text { if } u \neq v \text { and }(u, v) \in E(G) ; \\ \mu & \text { if } u \neq v \text { and }(u, v) \notin E(G) .\end{cases}
$$

Many generalizations of strongly regular graphs have been considered in the literature. For example, the $t$-tuple regular graphs were introduced by Cameron [2]; the NR-regular graphs (also known as the Gamma-Delta regular graphs) were defined by Godsil and McKay [5]; and the strong graphs were defined by Seidel [7]. Since every strongly regular graph has exactly three eigenvalues, graphs with few eigenvalues are also considered as generalizations of strongly regular graphs. In particular, graphs with three eigenvalues were studied by Van Dam [8]; graphs with three Laplacian eigenvalues were studied by Van Dam and Haemers [10]; and graphs with four eigenvalues were studied by Van Dam [9] and by Van Dam and Spence [11].

In this paper, we yet introduce another extension of strongly regular graphs, from a different direction. In 1966, Erdős, Rényi, and Sós [1,4] proved the following interesting result, commonly referred to as the Friendship Theorem: If $\delta(u, v)=1$ for any two distinct vertices $u, v$ in a graph $G$, then $G=K_{1} \vee\left(m K_{2}\right)$, where $m K_{n}$ denotes the disjoint union of $m$ copies of the complete graph on $n$ vertices. A nice interpretation of the theorem is that if any two people in a party have exactly one common friend, then there exists a politician who is a friend of everybody.

We generalize the Friendship Theorem as follows. Let $\lambda$ and $\mu$ be any nonnegative integers. A graph $G$ is call $a(\lambda, \mu)$-graph if every pair of adjacent vertices have $\lambda$ common neighbors, and every pair of non-adjacent vertices have $\mu$ common neighbors. Thus, $(\lambda, \mu)$-graphs are generalizations of strongly regular graphs obtained by relaxing the regularity property on vertex degrees. In particular, the Friendship Theorem asserts that $K_{1} \vee\left(m K_{2}\right)$ is the only type of $(1,1)$-graphs. Since strongly regular graphs have been studied extensively in the literature, we are interested in studying irregular $(\lambda, \mu)$-graphs. One might assume that there are many $(\lambda, \mu)$-graphs that are not regular (and thus not strongly regular). To the contrary, we prove that $K_{1} \vee\left(m K_{\lambda+1}\right)$ is the unique type of connected irregular $(\lambda, \mu)$-graphs. This extends the Friendship Theorem. As an immediate consequence, our result implies the following recent conjecture by Limaye, Sarvate, Stanica, and Young [6] on strongly bi-regular graphs. An $n$-vertex graph $G$ is called strongly bi-regular, denoted $S B R G(n, r, s, \lambda, \mu)$, if $G$ is not regular and there exist nonnegative integers $r, s, \lambda, \mu$ with $r \neq s$ such that for all vertices $u, v$,

$$
\delta(u, v)= \begin{cases}r \text { or } s & \text { if } u=v ; \\ \lambda & \text { if } u \neq v \text { and }(u, v) \in E(G) ; \\ \mu & \text { if } u \neq v \text { and }(u, v) \notin E(G) .\end{cases}
$$


Conjecture 1 (Limaye, Sarvate, Stanica, and Young, 2005). Let $G$ be a connected $S B R G(n, r, s, \lambda, \mu)$. Then $G=K_{1} \vee m K_{\lambda+1}$, where $n=m(\lambda+1)+1$.

\section{Characterization of Irregular $(\lambda, \mu)$-graphs}

In this section we prove that $K_{1} \vee m K_{\lambda+1}$ is the only type of connected irregular $(\lambda, \mu)$ graphs. A nice interpretation of the result is that if each pair of friends has exactly $\lambda$ common friends and each pair of strangers have exactly $\mu(\mu \geq 1)$ common friends in a party, then either everyone has exactly the same number of friends or there exists a politician who is everybody's friend.

Theorem 1. Suppose $G$ is an irregular $(\lambda, \mu)$-graph on $n$ vertices. Then one of the following is true:

i) $\mu=0$ and $G=m K_{\lambda+2}+t K_{1}$ (disjoint union of $m$ copies of $K_{\lambda+2}$ and $t$ copies of $\left.K_{1}\right)$, where $n=m(\lambda+2)+t$.

ii) $\mu=1$ and $G=K_{1} \vee\left(m K_{\lambda+1}\right)$, where $n=m(\lambda+1)+1$.

Proof. If $\mu=0$, then $G$ has no pair of vertices with distance two apart. Thus each component of $G$ is a complete graph. Since $G$ is a $(\lambda, \mu)$-graph, each complete of $G$ is either $K_{\lambda+2}$ or $K_{1}$; that is, $G=m K_{\lambda+2}+t K_{1}$. This proves part i) of the theorem.

Suppose now $\mu \neq 0$. For all distinct vertices $u, v$, define

$$
\epsilon(u, v)= \begin{cases}1 & \text { if }(u, v) \in E(G) ; \\ 0 & \text { if }(u, v) \notin E(G) .\end{cases}
$$

Claim 1: $(\mu-\epsilon(u, v))(d(u)-d(v))=0$ for any two distinct vertices $u, v$.

Proof of Claim 1. Let $\epsilon=\epsilon(u, v), A=N(u)-N(v)-\{v\}, B=N(u) \cap N(v)$, and $C=N(v)-N(u)-\{u\}$. Let $E(X, Y)$ be the set of edges with one vertex in $X$ and the other vertex in $Y$, and $E(x, Y)$ be the set of edges with one vertex being $x \in X$ and the other vertex in $Y$. Then, for each $b \in B$,

$$
\begin{aligned}
|E(b, A)| & = \begin{cases}|N(b) \cap N(u)|-|E(b, B)| & \text { if }(u, v) \notin E(G) ; \\
|N(b) \cap N(u)|-|E(b, B)|-1 & \text { if }(u, v) \in E(G) .\end{cases} \\
& =\lambda-|E(b, B)|-\epsilon
\end{aligned}
$$

and

$$
|E(B, A)|=\sum_{b \in B}|E(b, A)|=\sum_{b \in B}(\lambda-\epsilon)-\sum_{b \in B}|E(b, B)|=(\lambda-\epsilon)|B|-2|E(B, B)| .
$$

Similarly,

$$
|E(B, C)|=\sum_{b \in B}(|N(b) \cap N(v)|-|E(b, B)|-\epsilon)=(\lambda-\epsilon)|B|-2|E(B, B)| .
$$


Thus

$$
|E(A, B)|=|E(B, A)|=|E(B, C)|=|E(C, B)| .
$$

Next we count $|E(A, C)|$ in two different ways. First, for each $a \in A$, we have

$$
\begin{aligned}
|E(a, C)| & = \begin{cases}|N(a) \cap N(v)|-|E(a, B)| & \text { if }(u, v) \notin E(G) ; \\
|N(a) \cap N(v)|-|E(a, B)|-1 & \text { if }(u, v) \in E(G) .\end{cases} \\
& =\mu-|E(a, B)|-\epsilon .
\end{aligned}
$$

Thus

$$
|E(A, C)|=\sum_{a \in A}|E(a, C)|=\sum_{a \in A}(\mu-|E(a, B)|-\epsilon)=(\mu-\epsilon)|A|-|E(A, B)| .
$$

Similarly,

$$
|E(C, A)|=\sum_{c \in C}|E(c, A)|=\sum_{c \in C}(\mu-|E(c, B)|-\epsilon)=(\mu-\epsilon)|C|-|E(C, B)| .
$$

By (1),

$$
(\mu-\epsilon)|A|=|E(A, C)|+|E(A, B)|=|E(C, A)|+|E(C, B)|=(\mu-\epsilon)|C|
$$

and thus

$(\mu-\epsilon)(d(u)-d(v))=(\mu-\epsilon)((|A|+|B|+\epsilon)-(|C|+|B|+\epsilon))=(\mu-\epsilon)(|A|-|C|)=0$.

This completes the proof of Claim 1.

Recall that $\mu \neq 0$. Then $\mu=1$, since otherwise, by Claim $1, G$ would be regular. We now fix a vertex $u$ and define $V_{1}=\{v \in V: d(v)=d(u)\}$ and $V_{2}=\{v \in V: d(v) \neq d(u)\}$. Since $G$ is irregular, $V_{1}$ and $V_{2}$ are nonempty and form a partition for $V$.

Claim 2: Every vertex in $V_{1}$ is adjacent to every vertex in $V_{2}$.

Proof of Claim 2. Suppose otherwise $(x, y) \notin E(G)$ for some $x \in V_{1}$ and some $y \in V_{2}$. Then $\epsilon(x, y)=0$. By Claim 1, $d(x)=d(y)$, contracting the definition for $V_{1}$ and $V_{2}$. Claim 3: Either $\left|V_{1}\right|=1$ or $\left|V_{2}\right|=1$.

Proof of Claim 3. Suppose otherwise $\left|V_{1}\right| \geq 2$ and $\left|V_{2}\right| \geq 2$. Let $i=1,2$. If there were a pair of non-adjacent vertices $u, v$ in $V_{i}(i=1,2)$, then by Claim $2, \mu=\delta(u, v) \geq\left|V_{3-i}\right| \geq 2$, contradicting $\mu=1$. Thus there is no pair of non-adjacent vertices in each $V_{i}$. By Claim $2, G=K_{n}$, contradicting the irregularity of $G$.

By Claim 3, without loss of generality, let $V_{1}=\{u\}$. By Claim 2, $u$ is adjacent to every vertex in $V_{2}$. Thus $\mu=1$ implies that $G\left[V_{2}\right]$, the subgraph of $G$ induced by $V_{2}$, is a disjoint union of complete graphs $K_{\lambda}$. Therefore $G=K_{1} \vee\left(m K_{\lambda+1}\right)$ with $n=m(\lambda+1)+1$. This proves the theorem. 
Remark 1. Since strongly bi-regular graphs are a special case of irregular $(\lambda, \mu)$-graphs, Theorem 1 implies Conjecture 1 immediately.

Remark 2. We note that our definition of $(\lambda, \mu)$-graphs extends the concept of all three types of graphs discussed in [3]. Let $\bar{G}$ be the complement graph of $G$ and $N[u]=$ $N(u) \cup\{u\}$ be the closed neighborhood of a vertex $u$. It can easily be observed that a graph $G$ with $n$ vertices is uniformly $(2, r)$-regular (that is, $|N(u) \cup N(v)|=r$ for each pair of distinct vertices $u, v)$ iff $\bar{G}$ is an $(n-r-2, n-r)$-graph; $G$ is uniformly cl-nbhd $(2, r)$-regular (that is, $|N[u] \cup N[v]|=r$ for each pair of distinct vertices $u, v)$ iff $\bar{G}$ is an $(n-r, n-r)$-graph; $G$ is $k$-frienly (that is, $|N(u) \cap N(v)|=r$ for each pair of distinct vertices $u, v)$ iff $G$ is a $(k, k)$-graph. Therefore, Theorem 1 implies [3, Corollaries 1, 2, 3, 4].

Acknowledgement. We thank three referees for many valuable comments leading to the clear presentation of the paper.

\section{References}

[1] M. Aigner, G. Ziegler. Proofs from The Book. Springer-Verlag, Berlin, 1999.

[2] P. Cameron. Strongly regular graphs, Chapter 12 in Selected Topics in Graph Theory, pp. 337-360, L. Beineke and R. Wilson (Eds.), Academic Press, New York, 1978.

[3] K. Bragan, D. Hoffman, P. Johnson. Inferring regularity by a neglected theorem of Ryser. Bull. Inst. Combin. Appl. 50 (2007), 47-51.

[4] P. Erdős, A. Rényi, V. Sós. On a problem of graph theory. Studia Sci. Math. 1 (1966), 215-235.

[5] C.D. Godsil, B.D. MaKay. Graphs with regular neighborhoods. Combinatorial mathematics, VII (Proc. Seventh Australian Conf., Univ. Newcastle, Newcastle, 1979), pp. 127-140, Lecture Notes in Math., 829, Springer, Berlin-New York, 1980.

[6] N.B. Limaye, D.G. Sarvate, P. Stanica, P. Young. Regular and strongly regular planar graphs. J. Combin. Math. Combin. Comput. 54 (2005), 111-127.

[7] J.J. Seidel. Strongly regular graphs. 1969 Recent Progress in Combinatorics (Proc. Third Waterloo Conf. on Combinatorics, 1968), pp. 185-198, Academic Press, New York.

[8] E.R. van Dam. Nonregular graphs with three eigenvalues. J. Combin. Theory Ser. B 73 (1998), 101-118.

[9] E.R. van Dam. Regular graphs with four eigenvalues. Linear Algebra Appl. 226/228 (1995), 139-162.

[10] E.R. van Dam, W.H. Haemers. Graphs with constant $\mu$ and $\bar{\mu}$. Discrete Math. 182 (1998), no. 1-3, 293-307.

[11] E.R. van Dam, E. Spence. Small regular graphs with four eigenvalues. Discrete Math. 189 (1998), 233-257. 